\title{
The MIF rs755622 Variant may Increase Susceptibility of Breast Cancer but not Gastrointestinal Cancer in a Turkish Population
}

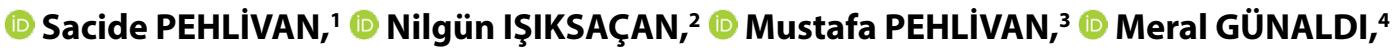 \\ (D) Yasemin OYACI, ${ }^{1}$ (D) Ayşe Feyda NURSAL ${ }^{5}$
}

'Department of Medical Biology, İstanbul University, İstanbul Faculty of Medicine, İstanbul-Turkey ${ }^{2}$ Department of Immunology, Dr. Sadi Konuk Training and Research Hospital, İstanbul-Turkey ${ }^{3}$ Department of Hematology, Gaziantep University, Faculty of Medicine, Gaziantep-Turkey ${ }^{4}$ Department of Oncology, Dr. Sadi Konuk Training and Research Hospital, İstanbul-Turkey ${ }^{5}$ Department of Medical Genetics, Hitit University, Faculty of Medicine, Çorum-Turkey

\begin{abstract}
OBJECTIVE
An increasing number of epidemiological and molecular evidence proposes that inflammation is a significant factor in the etiology of cancers. Macrophage Migration Inhibitory Factor (MIF) encodes a lymphokine involved in cell-mediated immunity, immunoregulation, and inflammation. It has been reported that MIF is linked with a higher risk of several cancer types. In the present study, we investigated the association of MIF rs755622 variant with the risk of breast cancer (BC) and gastrointestinal cancer in a Turkish cohort.

\section{METHODS}

The present study included a total of 153 subjects, which consisted of $33 \mathrm{BC}$ patients, 53 gastrointestinal cancer patients and 67 healthy controls. Genomic DNA extracted from peripheral venous blood. The rs755622 variant of the MIF gene was genotyped using polymerase chain reaction-restriction fragment length polymorphism (PCR-RFLP) method. The results were statistically analyzed by calculating the odds ratios (OR) and $95 \%$ confidence intervals $(\mathrm{CI})$ using the $\chi^{2}$ test.
\end{abstract}

\section{RESULTS}

There was a statistical difference between the BC patients and controls for the MIF rs755622 variant. MIF rs755622 GG genotype and G allele were increased in BC patients compared to controls ( $\mathrm{p}=0.016$, $\mathrm{p}=0.017$, respectively). No significant difference was observed between gastrointestinal cancer patients and controls for the MIF rs755622 variant ( $\mathrm{p}>0.05)$.

\section{CONCLUSION}

Our results showed that the MIF rs755622 variant might play a potential role in BC physiopathology.

Keywords: Breast cancer; gastrointestinal cancer; MIF gene; PCR-RFLP; variant.

Copyright $\odot$ 2020, Turkish Society for Radiation Oncology

\section{Introduction}

Breast cancer (BC) is the most common cancer and the leading cause of cancer mortality among women in the world.[1] Despite the decreased BC death rates owing to earlier diagnosis and better treatment modalities, the incidence of $\mathrm{BC}$ continues to increase in developing countries. $\mathrm{BC}$ is a multifactorial disease, and many- 
factors were linked with $\mathrm{BC}$ development and progress, such as polygenic inheritance, lifestyle, and exposure to radiation. Human immune responses include initiate and adaptive immune reactions, and their alterations may result in increased susceptibility to several diseases, such as cancer. Recent studies report strong evidence that cytokines and cells in the immune response are related to $\mathrm{BC}$ risk and prognosis.[2]

Gastrointestinal cancer is among the most common causes of mortality in the world. Many patients cannot be cured mainly due to late diagnosis, hence requiring palliative medical care. Patients' nutritional status, such as weight loss, muscle wasting known as sarcopenia, and inflammation, should be considered.[3] Systemic inflammation that arises from the tumor development and progression has been reported to play major roles in these adverse effects, and recent studies have found that some genetic polymorphisms involved in immune or inflammatory processes may have an impact on patient outcomes, including weight loss or survival, using the modulation of these pathways. [4]

Macrophage migration inhibitory factor (MIF) belongs to the transforming growth factor- $\beta$ (TGF- $\beta$ ) superfamily, which is considered a pleiotropic cytokine that is a key regulator of innate immunity. MIF serves as an upstream regulator of several other inflammatory cytokines. [5] The MIF gene, found on chromosome 22q11.2, belongs to the transforming growth factor-b (TGF-b) superfamily. It is synthesized by Tlymphocytes.[6] MIF is involved in inducing tumor growth, regulating immune responses, and facilitating tumor-associated angiogenesis. [7] It bears a single nucleotide polymorphism (SNP; G to C transition) located in the 5'-flanking region at position -173, which affects MIF gene expression.[8] Recent studies show that MIF variants play crucial roles in cancer susceptibility, including acute myeloid leukemia, colorectal cancer, bladder cancer, cervical cancer, acute lymphoblastic leukemia, gastric cancer, and prostate cancer.[9] However, to our knowledge, there have been no studies focusing on the MIF variants and the BC, gastrointestinal cancer risk in the Turkish population. In this study, we carried out this case-control research to evaluate the possible link between a common MIF rs755622 variant and BC, gastrointestinal cancer susceptibility in a Turkish population.

\section{Materials and Methods}

This study included a total 153 subjects,which consisted of $33 \mathrm{BC}$ patients and 53 gastrointestinal can- cer (consisting of gastric and colorectal cancers) patients and 67 healthy controls. All BC and gastrointestinal cancer tumors were histopathologically confirmed and the blood samples were recruited from the Department of Medical Oncology of of Dr. Sadi Konuk Training and Research Hospital (Istanbul, Turkey). The healthy control group was similar concerning age and sex distribution; subjects in this group did not have any evidence of any malignancy. Informed written consent was obtained from all participants. The study protocol was approved by the Local Ethics Committees in accordance with the ethical standard for human experimentation established by the Declaration of Helsinki.

\section{Genotyping}

Genomic DNA was extracted from peripheral blood leucocytes by the standard salting-out method.[10] PCR was performed using a forward (5'-ACTAAGAAAGACCCGAGGC-3') and reverse (5'-GGGGCACGTTGGTGTTTAC-3') primers. For MIF (-173), a 330 bp fragment was amplified, which was then digested with AluI restriction enzyme (Fermentas), overnight at $37^{\circ} \mathrm{C}$. The products were then separated on $3 \%$ agarose gel. The $330 \mathrm{bp} \mathrm{PCR} \mathrm{products}$ had a consistent restriction site resulting in 62 and 268 bp fragments. The GG genotype did not have a second cutting site for AluI. The CC genotype had a second cutting site, resulting in three fragments of size 205, 62 and $63 \mathrm{bp}$. As an internal quality control, to avoid sample or reading errors, the experiment was duplicated in $20 \%$ of the samples.[11]

\section{Statistical Analysis}

All statistical analyses were performed using the Statistical Package for the Social Sciencefor Windows (version 18.0; SPSS Inc, Chicago, IL, U.S.A.).The genotype distribution and allele frequency of the MIF gene rs755622 variant in control and patient groups were compared using the chi-square and Fisher's exact tests. Odds ratios (ORs) and 95\% confidence intervals (95\% CIs) were obtained using logistic regressions to investigate associations between genotype, allele distribution of MIF rs755622 variant and susceptibility of BC/Gastrointestinal cancer. The Hardy-Weinberg equilibrium (HWE) was calculated using the de Finetti program (Online HWE and Association Testing-InstitutfürHumangenetik, Munich, Germany). P value $\leq 0.05$ was accepted as statistically significant. 


\section{Results}

A case-control study was performed with a total of 153 subjects, inducting 86 cancer patients ( $33 \mathrm{BC}, 53$ gastrointestinal cancer) and 67 healthy controls. Clinical and demographical characteristics of BC patients (gender, age, weight, length, family history, smoking, menapause status, and molecular subtypes) are shown in Table 1.

The genotypic and allelic frequencies of MIF rs755622 are shown in Table 2. The genotype distribution of MIF rs755622 between BC patients and the control group had a significant difference. MIF rs755622 GG genotype was higher in BC patients than controls, while GC genotype was higher in control group com-

\begin{tabular}{lc}
\begin{tabular}{l} 
Table 1 \\
\multicolumn{1}{c}{$\begin{array}{c}\text { Baseline clinical and demographics features of } \\
\text { the BC patients }\end{array}$} \\
Characteristics
\end{tabular} \\
\hline Study group (n=33) \\
\hline Gender, female, $\mathrm{n}(\%)$ & $33(100)$ \\
Age, mean years & 60.33 \\
Weight, mean & 76.78 \\
Length, mean & 160.51 \\
Family history & $9(27.27)$ \\
Smoking & $9(27.27)$ \\
Menopause & $26(78.78)$ \\
Molecular subtypes & \\
Luminal A & $21(63.63)$ \\
Luminal B & $5(15.15)$ \\
HER2 (+) & $2(6.07)$ \\
Unknown & $5(15.15)$ \\
\hline
\end{tabular}

pared to BC patients $(\mathrm{p}=0.016$, and $\mathrm{p}=0.030$, respectively). MIF rs755622 $\mathrm{G}$ allele was more prevalent in $\mathrm{BC}$ patients compared to the control group $(\mathrm{p}=0.017)$. The genotype and allele frequencies of MIF rs755622 showed no statistically significant difference between gastrointestinal cancer patients and controls ( $\mathrm{p}>0.05)$. The genotypic frequencies for MIF rs755622 among patients and controls were in HWE ( $p=0.71$, and $=0.52$, respectively).

We investigated the association between $\mathrm{BC}$ molecular subtypes and MIF rs755622 genotype distribution. The patients with BC had Luminal A, Luminal B and HER2(+) molecular subtypes. There was no statistically significant association between BC molecular subtypes and genotype distribution (Table 3).

\section{Discussion}

Cancer remains to be a major cause of death worldwide. Various components of the innate immunity are induced in cancer pathogenesis to diminish cancer-mediated inflammation.[12] This process also induces adaptive immune responses for targeting cancer through more specific immune mechanisms. Macrophage migration inhibitory factor (MIF) was originally identified as a substance isolated from the supernatants of activated T lymphocyte culture and was manifested as a cytokine that can hinder the random migration of macrophages, being one of the first to be described.[13] Today, MIF is believed to be a multifunctional substance that induces the synthesis of inflammatory cytokines including tumor necrosis factor- $\alpha$

Table 2 The distribution of genotypes and alleles of MIF rs755622variant in groups

\begin{tabular}{|c|c|c|c|c|}
\hline MIF rs755622 & $\begin{array}{c}\text { BC patients } \\
n^{\mathrm{a}}=33(\%)\end{array}$ & $\begin{array}{l}\text { Gastrointestinal cancer patients } \\
\qquad n^{b}=53(\%)\end{array}$ & $\begin{array}{l}\text { Controls } \\
n=67(\%)\end{array}$ & $\mathbf{p}$ \\
\hline \multicolumn{5}{|l|}{ Genotypes } \\
\hline GG & $29(87.87)$ & 43 (81.14) & $44(65.67)$ & $\begin{array}{l}0.016^{a} \\
0.059^{b}\end{array}$ \\
\hline GC & $4(12.13)$ & 9 (16.98) & $21(31.34)$ & $\begin{array}{l}0.030^{\mathrm{a}} \\
0.064^{\mathrm{b}}\end{array}$ \\
\hline $\mathrm{CC}$ & $0(0)$ & $1(1.88)$ & $2(2.98)$ & $\begin{array}{l}0.255^{\mathrm{a}} \\
0.583^{\mathrm{b}}\end{array}$ \\
\hline \multicolumn{5}{|l|}{ Alleles } \\
\hline $\mathrm{G}$ & $62(93.93)$ & 95 (89.63) & 109 (81.34) & $\begin{array}{l}0.017^{\mathrm{a}} \\
0.074^{\mathrm{b}}\end{array}$ \\
\hline $\mathrm{C}$ & $4(6.07)$ & $11(10.37)$ & 25 (18.65) & \\
\hline HWEp & 0.71 & 0.52 & 0.78 & \\
\hline
\end{tabular}

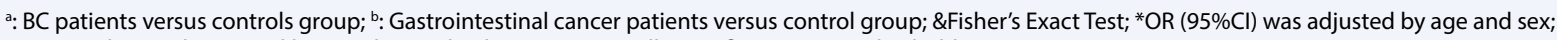
HWE: Hardy-Weinberg equilibrium. The results that are statistically significant are typed in bold 
Table 3 MIF genotype distribution according to $B C$ molecular subtypes

\begin{tabular}{|c|c|c|c|c|}
\hline \multirow[t]{2}{*}{ Molecular subtypes } & \multicolumn{2}{|c|}{ MIF rs755622 } & \multirow[b]{2}{*}{ Total } & \multirow[b]{2}{*}{$\mathbf{p}^{*}$} \\
\hline & $\begin{array}{c}\text { GG } \\
\text { n (\%) }\end{array}$ & $\begin{array}{c}\text { GC } \\
\text { n (\%) }\end{array}$ & & \\
\hline \multicolumn{5}{|l|}{ Luminal A } \\
\hline Yes & $20(95.24)$ & $1(4.76)$ & 21 & 0.125 \\
\hline No & $9(75)$ & $3(25)$ & 12 & \\
\hline \multicolumn{5}{|l|}{ Luminal B } \\
\hline Yes & $5(100)$ & $0(0)$ & 5 & 1 \\
\hline No & $23(82.14)$ & $5(17.86)$ & 28 & \\
\hline \multicolumn{5}{|l|}{ HER2 (+) } \\
\hline Yes & $2(100)$ & $0(0)$ & 2 & 1 \\
\hline No & $27(87.10)$ & $4(12.90)$ & 31 & \\
\hline
\end{tabular}

*: Fisher's exact test

(TNF- $\alpha$ ), interleukin-1 $\beta$ (IL-1 $\beta$ ), interleukin-6 (IL-6), and interferon (IFN- $\gamma)$.[13] Studies conducted since the discovery of MIF have supported its role in inflammation as well as in the innate and adaptive immune responses. [14] Therefore, because of its important role in the regulation of the inflammatory and immune responses, MIF has been considered to be the link that connects inflammatory response to cancer.[14] MIF also inhibits the pro-apoptotic and growth inhibitory function of the p53 tumor suppressor in neoplastic cell lines.[15] Breast, colon and lung-derived tumors all have significantly higher levels of MIF mRNA and/or protein compared to their noncancerous, normal tissue counterparts.[16] Notably, MIF overexpression in the serum of cancer patients and in tumor biopsies has been associated with increased tumor progression and metastasis. [17,18]

Polymorphisms with potential functional role have been described in the MIF gene promoter. SNP of the nucleotide position -173 ( $\mathrm{G}$ to $\mathrm{C}$ ) has been found to be related to modified levels of the MIF gene transcription in vitro.[19] Previous studies showed that the MIF rs755622 was linked with the risk of peptic ulcer disease, systemic lupus erythematosus, polycystic ovary syndrome and rheumatoid arthritis. [20] In addition, a growing number of evidence implied that MIF rs755622 was involved in the pathogenesis of cancer. Tong et al. found the MIF rs755622 could increase the risk of cancer among Asians but not in Caucasians.[20] Also, in a meta-analysis, findings showed that there is a significant relationship between having any $\mathrm{C}$ allele at the -173 site within the MIF promoter and cancer. [21] Ramireddy et al. reported that MIF rs755622 was related to acute myelocytic leukemia susceptibility in
Taiwan.[22] Yuan and colleagues reported that the MIF rs755622 could increase the risk of bladder cancer in southeast China.[23] It was reported that the MIF rs755622 might be associated with a higher risk of prostate cancer in Chinese.[24] Lin et al. reported that CG, CC, and CG-CC genotype carriers in MIF rs755622 have a significantly increased risk of $\mathrm{BC}$ in Chinese females.[9] In the present study, we found MIF rs755622 GG genotype and $G$ allele were more prevalent in $B C$ patients compared to the control group. However, GC genotype increased in control group than the BC patient group (Table 2). Because our findings are not compatible with other results, this may be due to the difference in lifestyles, as well as ethnic differences.We also evaluated the association of MIF rs755622 variant and molecular subtypes with the development of BC (Table 3). Investigating whether there is an association between MIF rs755622 and molecular subtypes in the BC patient group, we found no significant association ( $>0.05)$. Although it was not statistically significant, the MIF rs755622 GG genotype was more prevalent in all molecular subtypes.

It was reported that expression of the MIF was significantly linked with the location of gastric tumor. However, this expression has no statistically significant correlation with variables, including age, gender histological subtypes, distant metastasis, and lymph node involvement, stage and grade of the tumor.[25] He et al. analyzed tissue microarray containing 117 samples of gastric cancer and investigated adjacent non-cancer normal tissues for MIF expression by immunohistochemistry.[26] They found that MIF expression in gastric cancer tissues were higher than that in adjacent non-cancer normal tissues $(\mathrm{p}<0.001)$, and high level of 
MIF was associated with poor tumor differentiation, advanced tumor stage, lymph node metastasis, and poor patient survival ( $\mathrm{p}<0.05$ for all). There are not many studies examining the relationship between MIF rs755622 variant and gastrointestinal cancer.Li et al. found that subjects with MIF rs755622 GC genotype and $\mathrm{C}$ allele have an increased risk of severe chronic atrophic gastritis.[27] Also, they observed that the MIF rs755622 CC genotype and C allele increased intestinal metaplasia. It was found that MIF rs755622 was associated with colorectal cancer susceptibility in Taiwan. [28] In this study, there was no significant difference between genotype-allele frequencies of MIF rs755622 and gastrointestinal cancer risk in our population.

There are several limitations in our case-control analysis. The first limitationis that the study group is scarce in number and recruited from the same region. Secondly, other variations of the MIF gene have not been studied. Finally, MIF expression level was not evaluated.

\section{Conclusion}

Cancer is a multifactorial disease. Breast cancer (BC) and gastrointestinal cancer in the population probably result from complex interactions between many genetic and environmental factors over time. However, every finding obtained about this issue will help us to understand the molecular structure of the disease. In conclusion, this study suggested the MIF rs755622 may play a role in the etiology of BC in a Turkish population. Further studies with larger populations are needed to confirm our findings.

\section{Peer-review: Externally peer-reviewed.}

Conflict of Interest: The authors declare that they have no conflict of interest.

Ethics Committee Approval: The study protocol was approved by the Local Ethics Committees in accordance with the ethical standard for human experimentation established by the Declaration of Helsinki.

Financial Support: We do not have any financial support for this study.

Authorship contributions: Concept - S.P., M.G., N.I.; Design - S.P., M.P.; Supervision - M.P., S.P.; Funding - S.P.; Materials - N.I., M.G.; Data collection and/or processing None; Data analysis and/or interpretation - Y.O., D.K., S.P.; Literature search - A.F.N., D.K.; Writing - A.F.N., Y.O.; Critical review - A.F.N., S.P.

\section{References}

1. Siegel RL, Miller KD, Jemal A. Cancer statistics, 2016. CA Cancer J Clin 2016;66(1):7-30.

2. Pusztai L, Karn T, Safonov A, Abu-Khalaf MM, Bianchini G. New Strategies in Breast Cancer: Immunotherapy. Clin Cancer Res 2016;22(9):2105-10.

3. Toiyama Y, Miki C, Inoue Y, Tanaka K, Mohri Y, Kusunoki M. Evaluation of an inflammation-based prognostic score for the identification of patients requiring postoperative adjuvant chemotherapy for stage II colorectal cancer. Exp Ther Med 2011;2(1):95-101.

4. Tan BH, Ross JA, Kaasa S, Skorpen F, Fearon KC; European Palliative Care Research Collaborative. Identification of possible genetic polymorphisms involved in cancer cachexia: a systematic review. J Genet 2011;90(1):165-77.

5. Kim KW, Kim HR. Macrophage migration inhibitory factor: a potential therapeutic target for rheumatoid arthritis. Korean J Intern Med 2016;31(4):634-42.

6. O'Reilly C, Doroudian M, Mawhinney L, Donnelly SC. Targeting MIF in Cancer: Therapeutic Strategies, Current Developments, and Future Opportunities. Med Res Rev 2016;36(3):440-60.

7. Yasasever V, Camlica H, Duranyildiz D, Oguz H, Tas F, Dalay N. Macrophage migration inhibitory factor in cancer. Cancer Invest 2007;25(8):715-9.

8. Donn RP, Shelley E, Ollier WE, Thomson W; British Paediatric Rheumatology Study Group. A novel 5 '-flanking region polymorphism of macrophage migration inhibitory factor is associated with systemiconset juvenile idiopathic arthritis. Arthritis Rheum 2001;44(8):1782-5.

9. Lin S, Wang M, Liu X, Zhu W, Guo Y, Dai Z, et al. Association of genetic polymorphisms in MIF with breast cancer risk in Chinese women. Clin Exp Med 2017;17(3):395-401.

10. Miller SA, Dykes DD, Polesky HF. A simple salting out procedure for extracting DNA from human nucleated cells. Nucleic Acids Res 1988;16(3):1215.

11. Akcali A, Pehlivan S, Pehlivan M, Sever T, Neyal M. Association of macrophage migration inhibitory factor gene promoter polymorphisms with multiple sclerosis in Turkish patients. J Int Med Res 2010;38(1):69-77.

12. Chen DS, Mellman I. Oncology meets immunology: the cancer-immunity cycle. Immunity 2013;39(1):1-10.

13. Nobre CC, de Araújo JM, Fernandes TA, Cobucci RN, Lanza DC, Andrade VS, et al. Macrophage Migration Inhibitory Factor (MIF): Biological Activities and Relation with Cancer. Pathol Oncol Res 2017;23(2):235-44.

14. Babu SN, Chetal G, Kumar S. Macrophage migration inhibitory factor: a potential marker for cancer diagnosis and therapy. Asian Pac J Cancer Prev 2012;13(5):1737-44. 
15. Hudson JD, Shoaibi MA, Maestro R, Carnero A, Hannon GJ, Beach DH. A proinflammatory cytokine inhibits p53 tumor suppressor activity. J Exp Med 1999;190(10):1375-82.

16. Meyer-Siegler KL, Vera PL, Iczkowski KA, Bifulco C, Lee A, Gregersen PK, et al. Macrophage migration inhibitory factor (MIF) gene polymorphisms are associated with increased prostate cancer incidence. Genes Immun 2007;8(8):646-52.

17. Hira E, Ono T, Dhar DK, El-Assal ON, Hishikawa Y, Yamanoi A, et al. Overexpression of macrophage migration inhibitory factor induces angiogenesis and deteriorates prognosis after radical resection for hepatocellular carcinoma. Cancer 2005;103(3):588-98.

18. Kamimura A, Kamachi M, Nishihira J, Ogura S, Isobe $\mathrm{H}$, Dosaka-Akita $\mathrm{H}$, et al. Intracellular distribution of macrophage migration inhibitory factor predicts the prognosis of patients with adenocarcinoma of the lung. Cancer 2000;89(2):334-41.

19. Lang T, Foote A, Lee JP, Morand EF, Harris J. MIF: Implications in the Pathoetiology of Systemic Lupus Erythematosus. Front Immunol 2015;6:577.

20. Tong X, Zheng B, Tong Q, Liu S, Peng S, Yang X, et al. The MIF -173G/C gene polymorphism increase gastrointestinal cancer and hematological malignancy risk: evidence from a meta-analysis and FPRP test. Int J Clin Exp Med 2015;8(9):15949-57.

21. Vera PL, Meyer-Siegler KL. Association between macrophage migration inhibitory factor promoter region polymorphism $(-173 \mathrm{G} / \mathrm{C})$ and cancer: a metaanalysis. BMC Res Notes 2011;4:395.
22. Ramireddy L, Lin CY, Liu SC, Lo WY, Hu RM, Peng YC, et al. Association study between macrophage migration inhibitory factor-173 polymorphism and acute myeloid leukemia in Taiwan. Cell Biochem Biophys 2014;70(2):1159-65.

23. Yuan $Q$, Wang $M$, Wang $M$, Zhang $Z$, Zhang W. Macrophage migration inhibitory factor gene $-173 \mathrm{G}>\mathrm{C}$ polymorphism and risk of bladder cancer in southeast China: a case-control analysis. Mol Biol Rep 2012;39(3):3109-15.

24. Ding GX, Zhou SQ, Xu Z, Feng NH, Song NH, Wang $\mathrm{XJ}$, et al. The association between MIF-173 G>C polymorphism and prostate cancer in southern Chinese. J Surg Oncol 2009;100(2):106-10.

25. Nabizadeh Marvast M, Sima HR, Ghaffarzadehgan K, Taghizadeh Kermani A, Norouzi N. Clinicopathological significance of macrophage migration inhibitory factor and its relation with $\mathrm{p} 53$ in gastric cancer. J Gastrointest Cance. 2011;42(1):5-10.

26. He LJ, Xie D, Hu PJ, Liao YJ, Deng HX, Kung HF, et al. Macrophage migration inhibitory factor as a potential prognostic factor in gastric cancer. World J Gastroenterol. 2015 Sep 14;21(34):9916-26.

27. Li ZW, Wu Y, Sun Y, Liu LY, Tian MM, Feng GS, et al. Inflammatory cytokine gene polymorphisms increase the risk of atrophic gastritis and intestinal metaplasia. World J Gastroenterol 2010;16(14):1788-94.

28. Ramireddy L, Chen WT, Peng CT, Hu RM, Ke TW, Chiang HC, et al. Association Between Genetic Polymorphism of the MIF Gene and Colorectal Cancer in Taiwan. J Clin Lab Anal 2015;29(4):268-4. 